\title{
EXPANDED POLYSTYRENE FOAM AS A SUBSTITUTE FOR PLASTER OF PARIS FOR SPINAL SHELLS
}

\author{
D. R. GunN, Singapore
}

Professor of Orthopaedic Surgery, University of Singapore

Polystyrene foam is very light, easily shaped, durable, water-repellent, and not expensive. These properties suggested that it may be suitable as an alternative to plaster-of-Paris for making anterior and posterior shells for the treatment of spinal diseases.

Polystyrene foam is manufactured in Singapore and is available in blocks of a standard size, $4 \times 2 \times 1$ feet. The density of the material can be varied: upon expert advice it was decided to try out material with a density of two pounds per cubic foot.

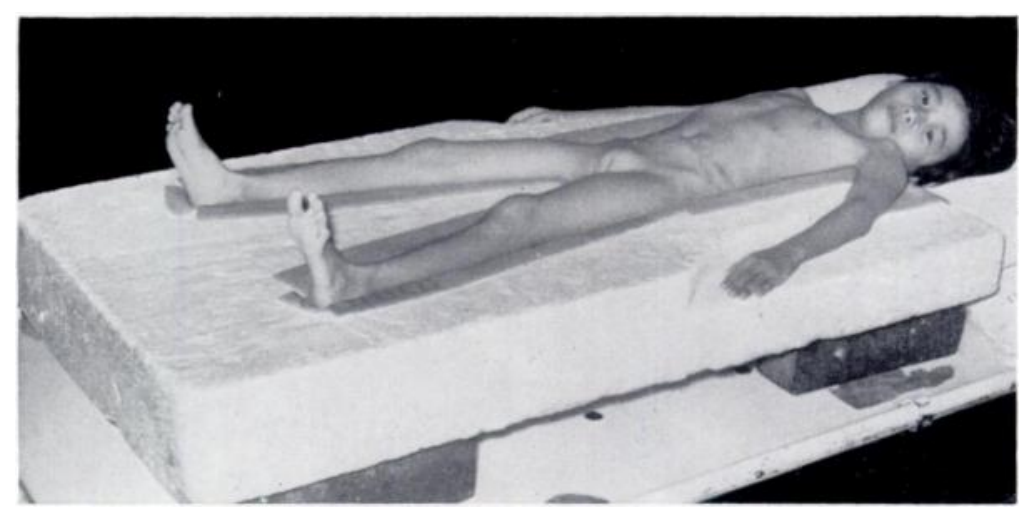

FIG. 1

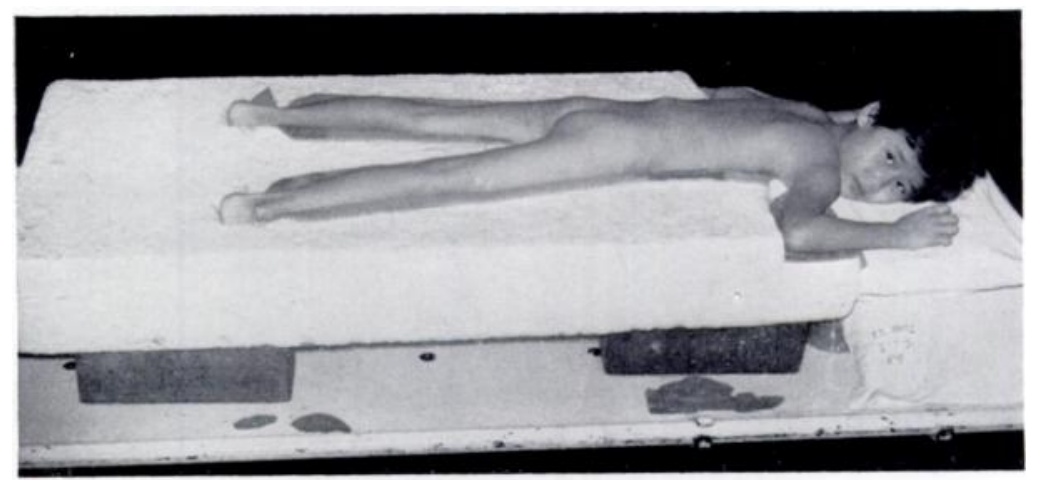

Fig. 2

Anterior and posterior shells.

It was thought that a six-inch thickness of polystyrene foam would be sufficient, so each slab was cut into two, six inches thick. The child was then laid on each slab in turn, and the anterior and posterior outlines were traced with a pencil. Each block was then hollowed out, with frequent fittings on the patient during the process (Figs. 1 to 3 ). It is estimated that the hollowing out and fitting of a shell takes one man one to two hours. 
When the shells were satisfactory they were lined with half-inch polyurethane foam: this is a cheap material that is available in cheerful colours. These shells are also being used for adult patients with satisfactory results.

After several months of usage the following advantages of this type of shell have become evident:

Comfort-The patients themselves like the polystyrene shells and say that they are comfortable: those who have had previous experience of plaster-of-Paris shells prefer polystyrene.

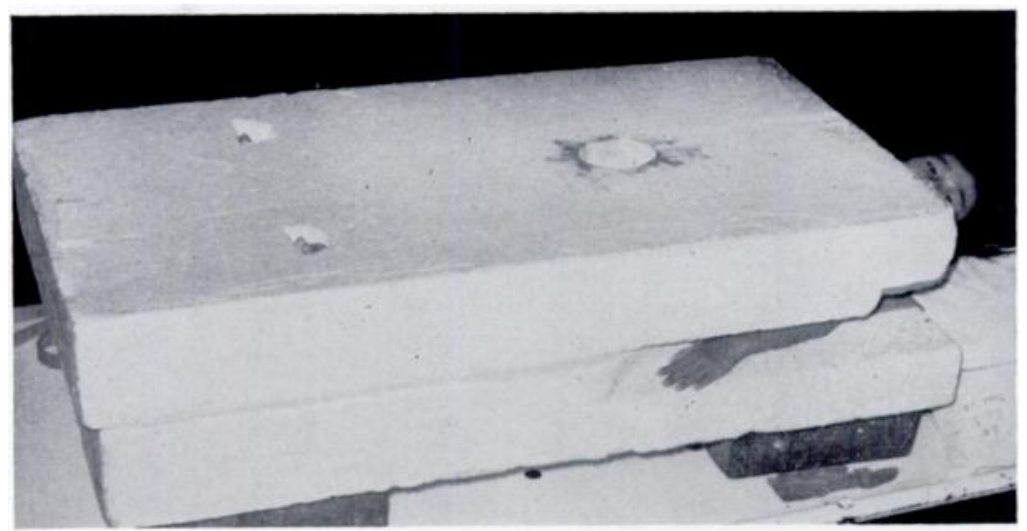

Fig. 3

Shells fitted together for turning.

Nursing - The shells are light and this fact is much appreciated by the nursing staff. Polystyrene is water-repellent, and staining and offensive odours from urine have not been apparent.

Durability-The material is strong and durable, and it seems possible that these shells may last a year or more. It is evident that they will last considerably longer than the average plaster-of-Paris shell.

Re-use-After use it is not difficult to cut out an inch or so from the top of the shell and to re-excavate the block to fit another child of approximately the same size.

Cost-Polystyrene is cheaper than plaster-of-Paris. One standard block, $4 \times 2 \times 1$ feet, will make one anterior and one posterior shell at the cost of approximately 60 per cent of the cost of plaster-of-Paris shells. The labour involved is much the same for either material. 